\title{
Stability Analysis of a Multigroup Epidemic Model with General Exposed Distribution and Nonlinear Incidence Rates
}

\author{
Ling Zhang, ${ }^{1}$ Jingmei Pang, ${ }^{2}$ and Jinliang Wang ${ }^{2}$ \\ ${ }^{1}$ School of Science, Department of Fundamental Mathematics, Jiamusi University, Jiamusi 154007, China \\ ${ }^{2}$ School of Mathematical Science, Heilongjiang University, Harbin 150080, China \\ Correspondence should be addressed to Jinliang Wang; jinliangwang@hit.edu.cn
}

Received 26 January 2013; Revised 16 June 2013; Accepted 22 July 2013

Academic Editor: Pagavathi Balasubramaniam

Copyright (c) 2013 Ling Zhang et al. This is an open access article distributed under the Creative Commons Attribution License, which permits unrestricted use, distribution, and reproduction in any medium, provided the original work is properly cited.

\begin{abstract}
We investigate a class of multigroup epidemic models with general exposed distribution and nonlinear incidence rates. For a simpler case that assumes an identical natural death rate for all groups, and with a gamma distribution for exposed distribution is considered. Some sufficient conditions are obtained to ensure that the global dynamics are completely determined by the basic production number $R_{0}$. The proofs of the main results exploit the method of constructing Lyapunov functionals and a graphtheoretical technique in estimating the derivatives of Lyapunov functionals.
\end{abstract}

\section{Introduction}

Multigroup epidemic models have been used in the literature to describe the transmission dynamics of many different infectious diseases such as mumps, measles, gonorrhea, HIV/AIDS and vector borne diseases such as Malaria [1]. In the models, heterogeneous host population can be divided into several homogeneous groups according to modes of transmission, contact patterns, or geographic distributions, so that within-group and intergroup interactions can be modeled separately. It is well known that global dynamics of multigroup models with higher dimensions, especially the global stability of the endemic equilibrium, are a very challenging problem. Guo et al. [2] proposed a graph-theoretic approach to the method of global Lyapunov functions and used it to resolve the open problem on the uniqueness and global stability of the endemic equilibrium of a multigroup SIR model with varying subpopulation sizes. Subsequently, a series of studies on the global stability of multigroup epidemic models were produced in the literature (see e.g., [2-5]).

In the present paper, a more general multigroup epidemic model is proposed and studied to describe the disease spread in a heterogeneous host population with general exposed distribution and nonlinear incidence rate. The host population is divided into $m$ distinct groups $(m \geq 1)$. For $1 \leq i \leq m$, the $i$ th group is further partitioned into four disjoint classes: the susceptible individuals, exposed individuals, infectious individuals, and recovered individuals, whose numbers of individuals at time $t$ are denoted by $S_{i}(t), E_{i}(t), I_{i}(t)$, and $R_{i}(t)$, respectively. Susceptible individuals infected with the disease but not yet infective are in the exposed (latent) class.

It is pointed in [6] that a fixed latent period can be considered as an approximation of the mean latent period, and this would be appropriate for those diseases whose latent periods vary only relatively slightly. For example, poliomyelitis has a latent period of 1-3 days (comparing to its much longer infectious period of 14-20 days). However disease such as tuberculosis, including bovine tuberculosis (a disease spread from animal to animal mainly by direct contact), may take months to develop to the infectious stage and also can relapse. Since the time it takes from the moment of new infection to the moment of becoming infectious may differ from disease to disease, even for the same disease, it differs from individual to individual, and it is indeed a random variable. It is thus of interest from both mathematical and biological viewpoints to investigate whether sustained oscillations are the result of general exposed distribution.

Following the method of [6], we also assume that the disease does not cause deaths during the latent period, taking the natural death rate into consideration. Let $P(t)$ denote 
the probability that an exposed individual remains in the time $t$ after entering the exposed class. For $1 \leq i, j \leq m$, $\beta_{i j} \geq 0$ denotes the coefficient of transmission between compartments $S_{i}$ and $I_{j}$. It is assumed that $m$-square matrix $\left(\beta_{i j}\right)_{1 \leq i, j \leq m}$ is irreducible [7]. So the proportion of exposed individuals can be expressed by the integral

$$
E_{i}(t)=\sum_{j=1}^{m} \beta_{i j} \int_{0}^{t} f_{i j}\left(S_{i}(u), I_{j}(u)\right) e^{-\delta_{j}(t-u)} P_{j}(t-u) d u
$$

where the sum takes into account cross-infections from all groups. Integrals in (1) are in the Riemann-Stieltjes sense. $P_{j}(t)$ satisfies the following reasonable properties:

(A) $P_{j}:[0, \infty) \rightarrow[0,1]$ is nonincreasing, piecewise continuous with possibly finitely many jumps and satisfies $P_{j}\left(0^{+}\right)=1$, and $\lim _{t \rightarrow \infty} P_{j}(t)=0$ with $\int_{0}^{\infty} P_{j}(t) d t$ is positive and finite.

Differentiating (1) gives

$$
\begin{aligned}
E_{i}^{\prime}(t)= & \sum_{j=1}^{m} \beta_{i j} f_{i j}\left(S_{i}(t), I_{j}(t)\right) \\
& +\sum_{j=1}^{m} \beta_{i j} \int_{0}^{t} f_{i j}\left(S_{i}(u), I_{j}(u)\right) e^{-\delta_{j}(t-u)} \\
& \times P_{j}^{\prime}(t-u) d u-\delta_{i} E_{i}(t)
\end{aligned}
$$

The first term on the right hand side in (2) is the rate at which new infected individuals come into the exposed class, and the last term explains the natural deaths. The second term accounts for the rate at which the individuals move to the infectious class (noting that $P_{j}^{\prime}(t-u) \leq 0$ due to the aformentioned property) from the exposed class; hence

$$
\begin{aligned}
I_{i}^{\prime}(t)= & -\sum_{j=1}^{m} \beta_{i j} \int_{0}^{t} f_{i j}\left(S_{i}(u), I_{j}(u)\right) e^{-\delta_{j}(t-u)} \\
& \times P_{j}^{\prime}(t-u) d u-\left(\delta_{i}+\varepsilon_{i}+\gamma_{i}\right) I_{i}(t) .
\end{aligned}
$$

Let $h_{j}(t)=-P_{j}^{\prime}(t)$ be the probability density function for the time (a random variable) it takes for an infected individual in the ith group to become infectious. Then (4) becomes

$$
\begin{aligned}
I_{i}^{\prime}(t)= & \sum_{j=1}^{m} \beta_{i j} \int_{0}^{t} f_{i j}\left(S_{i}(u), I_{j}(u)\right) e^{-\delta_{j}(t-u)} \\
& \times h_{j}(t-u) d u-\left(\delta_{i}+\varepsilon_{i}+\gamma_{i}\right) I_{i}(t) .
\end{aligned}
$$

Within the $i$ th group, $\varphi_{i}\left(S_{i}\right)$ denotes the growth rate of $S_{i}$, which includes both the production and the natural death of susceptible individuals. Therefore, under the assumptions, the model to be studied takes the following differential and integral equations form:

$$
\begin{aligned}
S_{i}^{\prime}(t)= & \varphi_{i}\left(S_{i}(t)\right)-\sum_{j=1}^{m} \beta_{i j} f_{i j}\left(S_{i}(t), I_{j}(t)\right), \\
E_{i}^{\prime}(t)= & \sum_{j=1}^{m} \beta_{i j} f_{i j}\left(S_{i}(t), I_{j}(t)\right) \\
& -\sum_{j=1}^{m} \beta_{i j} \int_{0}^{t} f_{i j}\left(S_{i}(u), I_{j}(u)\right) e^{-\delta_{j}(t-u)} \\
& \times h_{j}(t-u) d u-\delta_{i} E_{i}(t), \\
I_{i}^{\prime}(t)= & \sum_{j=1}^{m} \beta_{i j} \int_{0}^{t} f_{i j}\left(S_{i}(u), I_{j}(u)\right) e^{-\delta_{j}(t-u)} \\
& \times h_{j}(t-u) d u-\left(\delta_{i}+\varepsilon_{i}+\gamma_{i}\right) I_{i}(t), \\
& R_{i}^{\prime}(t)=\gamma_{i} I_{i}(t)-\delta_{i} R_{i}(t) .
\end{aligned}
$$

Since the variables $E_{i}$ and $R_{i}$ do not appear in the first and third equations of model (5), $E_{i}(t)$ and $R_{i}(t), i=1, \ldots, m$, can be decoupled from the $S_{i}(t)$ and $I_{i}(t)$ equations; we only need to consider the subsystem of (5) consisting of only the $S_{i}$ and $I_{i}$ equations:

$$
\begin{aligned}
S_{i}^{\prime}(t)= & \varphi_{i}\left(S_{i}(t)\right)-\sum_{j=1}^{m} \beta_{i j} f_{i j}\left(S_{i}(t), I_{j}(t)\right), \\
I_{i}^{\prime}(t)= & \sum_{j=1}^{m} \beta_{i j} \int_{0}^{t} f_{i j}\left(S_{i}(u), I_{j}(u)\right) e^{-\delta_{j}(t-u)} \\
& \times h_{j}(t-u) d u-\left(\delta_{i}+\varepsilon_{i}+\gamma_{i}\right) I_{i}(t),
\end{aligned}
$$

where $\delta_{i}$ denotes the natural death rates of $I_{i}$ compartments in the $i$ th group, $\varepsilon_{i}$ is the death rate caused by disease in the $i$ th group, and $\gamma_{i}$ is the rate of recovery of infectious individuals in the $i$ th group. In what follows we investigate the global stability of system (5).

When $m=1, P(t)=e^{\epsilon t}$, and with bilinear incidence rate, system (5) will reduce to the standard SEIR ordinary differential equation (ODE) model studied in $[8,9]$, and if we further assume that $P(t)$ is a step function, system (5) becomes the SEIR model with a discrete delay studied in [10]. Recently, a model of this type, but including the possibility of disease relapse, has been proposed in $[11,12]$ to investigate the transmission of herpes, and its global dynamics have been completely investigated in $[5,13]$.

To express the main idea and the approaches more clearly, we consider a simpler case in which all groups share the same natural death rate: $\delta_{j}=\delta$ for $j=1,2, \ldots, m$. Further, we assume that the functions $h_{j}(u)$ are disease specific only, 
implying that $h_{j}(u)=h(u)$ for $j=1,2, \ldots, m$. We choose the gamma distribution:

$$
h(u)=h_{n, b}(u)=\frac{u^{n-1}}{(n-1) ! b^{n}} e^{-u / b},
$$

where $b>0$ is a real number and $n>1$ is an integer, which is widely used and can approximate several frequently used distributions. For example, when $b \rightarrow 0^{+}, h_{n, b}(s)$ will approach the Dirac delta function, and when $n=1, h_{n, b}(s)$ is an exponentially decaying function.

The main object of this paper is to carry out the wellknown "linear chain trick" to system (6), transfer system into an equivalent ordinary differential equations system, and establish its global dynamics. We derive the basic reproductive number $R_{0}$ and show that $R_{0}$ completely determines the global dynamics of system (6). More specifically, if $R_{0} \leq 1$, the disease-free equilibrium is globally asymptotically stable and the disease dies out; if $R_{0}>1$, a unique endemic equilibrium exists and is globally asymptotically stable, and the disease persists at the endemic equilibrium. The global stability of $P^{*}$ rules out any possibility for Hopf bifurcations and the existence of sustained oscillations. We should point out here that this work is motivated by Yuan and Zou $[11,12,14]$. In the proof we demonstrate that the graph-theoretic approach developed in $[2,3]$ can be successfully applied to construct suitable Lyapunov functionals and thus prove the global stability of the endemic equilibrium for model (6) with general exposed distribution and nonlinear incidence rate. Our work is also based on a recent work by Sun and Shi [15], which resolved the dynamics of multigroup SEIR epidemic models with nonlinear incidence of infection and nonlinear removal functions between compartments.

In Section 2, we first give the model, preliminaries and the basic reproduction number $R_{0}$. The global stability of the corresponding equilibria for $R_{0} \leq 1$ and $R_{0}>1$ is shown, respectively, in Section 3-the key results of this paper. And in Section 4, some numerical simulations are shown to illustrate the effectiveness of the proposed result.

\section{Preliminaries}

We make the following basic assumptions for the intrinsic growth rate of susceptible individuals in the $i$ th group $\varphi_{i}\left(S_{i}\right)$ and the transmission functions $f_{i j}\left(S_{i}, I_{j}\right)$.

$\left(A_{1}\right) \varphi_{i}$ are $C^{1}$ non-increasing functions on $[0, \infty)$ with $\varphi_{i}(0)>0$, and there is a unique positive solution $\xi=S_{i}^{0}$ for the equation $\varphi_{i}(\xi)=0 . \varphi_{i}(S)>0$ for $0 \leq S<S_{i}^{0}$, and $\varphi_{i}(S)<0$ for $S>S_{i}^{0}$; that is

$$
\begin{aligned}
& {\left[\varphi_{i}\left(S_{i}\right)-\varphi_{i}\left(S_{i}^{0}\right)\right]\left(S_{i}-S_{i}^{0}\right)<0,} \\
& \text { for } S_{i} \neq S_{i}^{0}, i=1,2, \ldots, m
\end{aligned}
$$

$$
\begin{aligned}
& \left(A_{2}\right) f_{i j}\left(S_{i}, I_{j}\right) \leq c_{i j}\left(S_{i}\right) I_{j} \text { for all } I_{j}>0 . \\
& \left(A_{3}\right) c_{i j}\left(S_{i}\right) \leq c_{i j}\left(S_{i}^{0}\right), 0<S_{i}<S_{i}^{0}, i, j=1, \ldots, m .
\end{aligned}
$$

Following the technique and method in [14], define

$$
\widehat{b} \equiv \frac{b}{1+\delta b}
$$

which can absorb the exponential term $e^{-\delta u}$ into the delay kernel. The second equation in (6) can be rewritten as

$$
\begin{aligned}
I_{i}^{\prime}(t)= & \sum_{j=1}^{m} \frac{\beta_{i j}}{(1+\delta b)^{n}} \int_{0}^{t} f_{i j}\left(S_{i}, I_{j}\right) h_{n, \widehat{b}}(t-u) d u \\
& -\left(\delta+\varepsilon_{i}+\gamma_{i}\right) I_{i} .
\end{aligned}
$$

For $l=1, \ldots, n$, let

$$
\begin{array}{r}
y_{i, l}(t)=\sum_{j=1}^{m} \frac{\beta_{i j} \widehat{b}}{(1+\delta b)^{n}} \int_{0}^{t} f_{i j}\left(S_{i}, I_{j}\right) h_{l, \widehat{b}}(t-u) d u, \\
i=1,2, \ldots, m .
\end{array}
$$

Thus, for $l \in\{2, \ldots, n\}$, we obtain

$$
\begin{aligned}
\dot{y}_{i, l}= & h_{l, \widehat{b}}(0) \sum_{j=1}^{m} \frac{\beta_{i j} \widehat{b}}{(1+\delta b)^{n}} f_{i j}\left(S_{i}, I_{j}\right) \\
& +\sum_{j=1}^{m} \frac{\beta_{i j} \widehat{b}}{(1+\delta b)^{n}} \int_{-\infty}^{t} \frac{(l-1)(t-u)^{l-2}}{(l-1) ! \widehat{b}^{l}} e^{-(t-u) / \widehat{b}} f_{i j}\left(S_{i}, I_{j}\right) d u \\
& -\sum_{j=1}^{m} \frac{\beta_{i j} \widehat{b}}{(1+\delta b)^{n}} \int_{-\infty}^{t} \frac{(t-u)^{l-1}}{(l-1) ! \widehat{b}^{l+1}} e^{-(t-u) / \widehat{b}} f_{i j}\left(S_{i}, I_{j}\right) d u \\
= & \frac{\left[y_{i, l-1}-y_{i, l}\right]}{\widehat{b}} .
\end{aligned}
$$

For $l=1$, we have

$$
\begin{array}{r}
y_{i, 1}=\sum_{j=1}^{m} \frac{\beta_{i j} \widehat{b}}{(1+\delta b)^{n}} \int_{-\infty}^{t} \frac{e^{-(t-u) / \widehat{b}}}{\widehat{b}} f_{i j}\left(S_{i}, I_{j}\right) d u \\
i=1, \ldots, m .
\end{array}
$$

It follows that

$$
\begin{aligned}
\dot{y}_{i, 1}= & \sum_{j=1}^{m} \frac{\beta_{i j}}{(1+\delta b)^{n}} f_{i j}\left(S_{i}, I_{j}\right) \\
& -\sum_{j=1}^{m} \frac{\beta_{i j}}{(1+\delta b)^{n}} \int_{-\infty}^{t} \frac{e^{-(t-u) / \widehat{b}}}{\widehat{b}} f_{i j}\left(S_{i}, I_{j}\right) d u \\
= & \sum_{j=1}^{m} \frac{\beta_{i j}}{(1+\delta b)^{n}} f_{i j}\left(S_{i}, I_{j}\right)-\frac{1}{\widehat{b}} y_{i, 1}, \quad i=1, \ldots, m .
\end{aligned}
$$


Thus the integro-differential system (6) is equivalent to the ordinary differential equations

$$
\begin{gathered}
S_{i}^{\prime}(t)=\varphi_{i}\left(S_{i}(t)\right)-\sum_{j=1}^{m} \beta_{i j} f_{i j}\left(S_{i}(t), I_{j}(t)\right), \\
y_{i, 1}^{\prime}(t)=\frac{1}{(1+\delta b)^{n}} \sum_{j=1}^{m} \beta_{i j} f_{i j}\left(S_{i}(t), I_{j}(t)\right)-\frac{1}{\widehat{b}} y_{i, 1}(t), \\
y_{i, 2}^{\prime}(t)=\frac{1}{\widehat{b}}\left(y_{i, 1}(t)-y_{i, 2}(t)\right), \quad i=1,2, \ldots, m, \\
\vdots \\
y_{i, n}^{\prime}(t)=\frac{1}{\widehat{b}}\left(y_{i, n-1}(t)-y_{i, n}(t)\right), \\
I_{i}^{\prime}(t)=\frac{1}{\widehat{b}} y_{i, n}(t)-\left(\delta+\varepsilon_{i}+\gamma_{i}\right) I_{\mathrm{i}}(t) .
\end{gathered}
$$

For initial condition

$$
\begin{aligned}
& \left(S_{1}(0), y_{1,1}(0), \ldots, y_{1, n}(0), I_{1}(0)\right. \\
& \quad S_{2}(0), y_{2,1}(0), \ldots, y_{1, n}(0), I_{2}(0), \ldots, \\
& \left.\quad S_{m}(0), y_{m, 1}(0), \ldots, y_{m, n}(0), I_{m}(0)\right) \in \mathrm{R}^{m(n+2)},
\end{aligned}
$$

the existence, uniqueness, and continuity of the solution $\left(S_{i}, y_{i, 1}, y_{i, 2}, \ldots, y_{i, n}, I_{i}\right)$ of system (15) follow from the standard theory of Volterra integro-differential equation [16]. It can also be verified that every solution of (15) with nonnegative initial condition remains nonnegative.

It follows from $\left(A_{1}\right)$ and the first equation of (15) that $\lim \sup _{t \rightarrow \infty} S_{i}(t) \leq S_{i}^{0}$ for all $i=1,2, \ldots, m$. Let $N_{\varphi_{i}}$ be the maximum of the function $\varphi_{i}$ on $\mathrm{R}_{+}$and let $q$ be a positive real number such that $q>\widehat{b} N_{\varphi_{i}}$. Denote by $\Upsilon_{i}$ the $i$ th tube for system (15); that is,

$$
\Upsilon_{i}=\left(S_{i}, y_{i, 1}, y_{i, 2}, \ldots, y_{i, n}, I_{i}\right)
$$

It follows from a similar argument to that in [14] that we can show that the set $D_{\epsilon}$ defined by

$$
\begin{gathered}
\Gamma_{\epsilon}=\left\{\left(S_{i}, y_{i, 1}, y_{i, 2}, \ldots, y_{i, n}, I_{i}\right) \in \mathrm{R}_{+}^{m(n+2)}\right. \\
S_{i} \leq S_{i}^{0}+\epsilon, S_{i}+(1+\delta b)^{n} y_{i, 1} \leq q+S_{i}^{0}, \\
y_{i, l} \leq \frac{q+S_{i}^{0}+l \epsilon}{(1+\delta b)^{n}}, \\
I_{i} \leq \frac{q+S_{i}^{0}+(n+1) \epsilon}{\widehat{b}(1+\delta b)^{n}\left(\delta+\epsilon_{i}+\gamma_{i}\right)}, \\
i=1,2, \ldots, m, l=2,3, \ldots, n\}
\end{gathered}
$$

is a forward invariant compact absorbing set for system (15) for $\epsilon>0$ and that the set $\Gamma_{0}$ (i.e., when $\epsilon=0$ ) is a forward invariant compact set.
Under the assumption $\left(A_{1}\right)$, we know that system (15) always has the disease-free equilibrium

$$
\begin{aligned}
P_{0} & =\left(S_{1}^{0}, 0, \ldots, 0, I_{1}^{0}, S_{2}^{0}, 0, \ldots, 0, I_{2}^{0}, \ldots, S_{m}^{0}, 0, \ldots, 0, I_{m}^{0}\right) \\
& \in \mathrm{R}^{m(n+2)} .
\end{aligned}
$$

An equilibrium $P^{*}$ of (6) has the form $P^{*}=$ $\left(S_{1}^{*}, I_{1}^{*}, S_{2}^{*}, I_{2}^{*}, \ldots, S_{m}^{*}, I_{m}^{*}\right) \in \mathrm{R}^{2 m}$ with $S_{i}^{*}>0, I_{i}^{*}>0$, $i=1, \ldots, m$. Translating to the equivalent system (15), $P^{*}$ is corresponding to

$$
\begin{gathered}
\bar{P}^{*}=\left(S_{1}^{*}, y_{1,1}^{*}, \ldots, y_{1, n}^{*}, I_{1}^{*}, S_{2}^{*}, y_{2,1}^{*}, \ldots, y_{2, n}^{*}, I_{2}^{*}, \ldots,\right. \\
\left.S_{m}^{*}, y_{m, 1}^{*}, \ldots, y_{m, n}^{*}, I_{m}^{*}\right) \in \mathrm{R}^{m(n+2)} .
\end{gathered}
$$

$\bar{P}^{*}$ in the interior of $\Gamma_{0}$ is called an endemic equilibrium, and it satisfies the following equilibrium equations:

$$
\begin{gathered}
0=\varphi_{i}\left(S_{i}^{*}\right)-\sum_{j=1}^{m} \beta_{i j} f_{i j}\left(S_{i}^{*}, I_{j}^{*}\right), \\
0=\frac{1}{(1+\delta b)^{n}} \sum_{j=1}^{m} \beta_{i j} f_{i j}\left(S_{i}^{*}, I_{j}^{*}\right)-\frac{1}{\widehat{b}} y_{i, 1}^{*}, \\
0=\frac{1}{\widehat{b}}\left(y_{i, 1}^{*}-y_{i, 2}^{*}\right), \\
\vdots \\
0=\frac{1}{\widehat{b}}\left(y_{i, n-1}^{*}-y_{i, n}^{*}\right), \\
0=\frac{1}{\widehat{b}} y_{i, n}^{*}-\left(\delta+\varepsilon_{i}+\gamma_{i}\right) I_{i}^{*} .
\end{gathered}
$$

The basic reproduction number $R_{0}$ is defined as the expected number of secondary cases produced by single infectious individual during its entire period of infectiousness in a completely susceptible population. For system (15), we can calculate it as the spectral radius of a matrix called the next generation matrix. Let

$$
\begin{aligned}
& \mathscr{F}=\left(\begin{array}{ccc}
\frac{c_{11}\left(S_{1}^{0}\right) \beta_{11}}{(1+\delta b)^{n}} & \cdots & \frac{c_{1 m}\left(S_{1}^{0}\right) \beta_{1 m}}{(1+\delta b)^{n}} \\
\vdots & \ddots & \vdots \\
\frac{c_{m 1}\left(S_{m}^{0}\right) \beta_{m 1}}{(1+\delta b)^{n}} & \cdots & \frac{c_{m m}\left(S_{m}^{0}\right) \beta_{m m}}{(1+\delta b)^{n}}
\end{array}\right), \\
& \mathscr{V}=\operatorname{diag}\left(\delta+\varepsilon_{i}+\gamma_{i}\right) \\
& =\left(\begin{array}{cccc}
\delta+\varepsilon_{1}+\gamma_{1} & 0 & \cdots & 0 \\
0 & \delta+\varepsilon_{2}+\gamma_{2} & \cdots & 0 \\
\vdots & \vdots & \ddots & \vdots \\
0 & 0 & \cdots & \delta+\varepsilon_{m}+\gamma_{m}
\end{array}\right) .
\end{aligned}
$$


Then the next generation matrix is

$$
\mathscr{F} \mathscr{V}^{-1}=\left(\begin{array}{ccc}
\frac{c_{11}\left(S_{1}^{0}\right) \beta_{11}}{(1+\delta b)^{n}\left(\delta+\varepsilon_{1}+\gamma_{1}\right)} & \cdots & \frac{c_{1 m}\left(S_{1}^{0}\right) \beta_{1 m}}{(1+\delta b)^{n}\left(\delta+\varepsilon_{m}+\gamma_{m}\right)} \\
\vdots & \ddots & \vdots \\
\frac{c_{m 1}\left(S_{1}^{0}\right) \beta_{m 1}}{(1+\delta b)^{n}\left(\delta+\varepsilon_{1}+\gamma_{1}\right)} & \cdots & \frac{c_{m m}\left(S_{1}^{0}\right) \beta_{m m}}{(1+\delta b)^{n}\left(\delta+\varepsilon_{m}+\gamma_{m}\right)}
\end{array}\right),
$$

and hence, the basic reproduction number $R_{0}$ is

$$
R_{0}=\rho\left(\mathscr{F} \mathscr{V}^{-1}\right)=\max \left\{|\lambda| ; \lambda \in \sigma\left(\mathscr{F} \mathscr{V}^{-1}\right)\right\}
$$

where $\rho(\cdot)$ and $\sigma(\cdot)$ denote the spectral radius and the set of eigenvalues of a matrix, respectively. Since it can be verified that system $(15)$ satisfies conditions $\left(A_{1}\right)-\left(A_{5}\right)$ of Theorem 2 of [17], we have the following proposition.

Lemma 1. For system (15), the disease-free equilibrium $P_{0}$ is locally asymptotically stable if $R_{0}<1$, while it is unstable if $R_{0}>1$.

Following the method of [2], one defines a matrix

$$
\begin{aligned}
M^{0} & =\mathscr{V}^{-1} \mathscr{F} \\
& =\left(\begin{array}{ccc}
\frac{c_{11}\left(S_{1}^{0}\right) \beta_{11}}{(1+\delta b)^{n}\left(\delta+\varepsilon_{1}+\gamma_{1}\right)} & \cdots & \frac{c_{1 m}\left(S_{1}^{0}\right) \beta_{1 m}}{(1+\delta b)^{n}\left(\delta+\varepsilon_{1}+\gamma_{1}\right)} \\
\vdots & \ddots & \vdots \\
\frac{c_{m 1}\left(S_{1}^{0}\right) \beta_{m 1}}{(1+\delta b)^{n}\left(\delta+\varepsilon_{m}+\gamma_{m}\right)} & \cdots & \frac{c_{m m}\left(S_{1}^{0}\right) \beta_{m m}}{(1+\delta b)^{n}\left(\delta+\varepsilon_{m}+\gamma_{m}\right)}
\end{array}\right),
\end{aligned}
$$

whose spectral radius has a similar threshold property to that of $R_{0}$, since both of the nonnegative matrices $\mathscr{F} \mathscr{V}^{-1}$ and $M^{0}$ are irreducible, and hence from the Perron-Frobenius theorem [7] that their spectral radii are given by each of their simple eigenvalues. Thus, we obtain $R_{0}=\rho\left(\mathscr{F} \mathscr{V}^{-1}\right)=$ $\rho\left(\mathscr{V}^{-1} \mathscr{F}\right)=\rho\left(M^{0}\right)$. Then the following lemma immediately follows.

Lemma 2. $\rho\left(M^{0}\right) \leq 1$ if and only if $R_{0} \leq 1$.

\section{Main Results}

The following main theorems are summarized in terms of system (15).

Theorem 3. Assume that the functions $\varphi_{i}$ and $f_{i j}$ satisfy assumptions $\left(A_{1}\right)-\left(A_{3}\right)$, and the matrix $B=\left(\beta_{i j}\right)_{m \times m}$ is irreducible and $R_{0}$ is defined by (24).

(i) If $R_{0} \leq 1$, then $P_{0}$ is the unique equilibrium of system (15), and $P_{0}$ is globally asymptotically stable in $\Gamma_{0}$.

(ii) If $R_{0}>1$, then $P_{0}$ is unstable, and system (15) is uniformly persistent in $\Gamma_{0}$.
Proof. Let us define $M(S)=\left(\beta_{i j} c_{i j}\left(S_{i}\right) /(1+\delta b)^{n}\right.$ $\left.\left(\delta+\varepsilon_{i}+\gamma_{i}\right)\right)_{m \times m}$, where $S=\left(S_{1}, S_{2}, \ldots, S_{m}\right)^{T}$. Note that $M\left(S_{0}\right)=M^{0}$. Since $B=\left(\beta_{i j}\right)_{m \times m}$ is irreducible, the matrix $M^{0}$ is also irreducible.

First we claim that there does not exist any endemic equilibrium $\bar{P}^{*}$ in $\Omega$. Suppose that $S \neq S_{0}$. Then we have $0<M(S)<M^{0}$. Since nonnegative matrix $M(S)+M^{0}$ is irreducible, it follows from the Perron-Frobenius theorem (see Corollary 2.1.5 of [7]) that $\rho(M(S))<\rho\left(M^{0}\right) \leq 1$. This implies that $M(S) I=I$ has only the trivial solution $I=0$, where $I=\left(I_{1}, \ldots, I_{m}\right)^{T}$. Hence the claim is true. Next we claim that the disease-free equilibrium $P_{0}$ is globally asymptotically stable in $\Gamma_{0}$. From the Perron-Frobenius (see Theorem 2.1.4 of [7]), the nonnegative irreducible matrix $M^{0}$ has a strictly positive left eigenvector $\left(\omega_{1}, \omega_{2}, \ldots, \omega_{m}\right)$ associated with the eigenvalue $\rho\left(M^{0}\right)$ such that

$$
\left(\omega_{1}, \omega_{2}, \ldots, \omega_{m}\right) \rho\left(M^{0}\right)=\left(\omega_{1}, \omega_{2}, \ldots, \omega_{m}\right) M^{0} .
$$

Using this positive eigenvector, we construct the following Lyapunov function:

$$
V_{\mathrm{DFE}}=\sum_{i=1}^{m} \frac{\omega_{i}}{\delta+\varepsilon_{i}+\gamma_{i}}\left(\sum_{j=1}^{n} y_{i, j}+I_{i}\right) .
$$

Computing the derivative of $V_{\mathrm{DFE}}$ along the solutions of (15) in $\Gamma_{0}$, we get

$$
\begin{aligned}
V_{\mathrm{DFE}}^{\prime} & =\sum_{i=1}^{m}\left[\sum_{j=1}^{m} \frac{\omega_{i} \beta_{i j}}{(1+\delta b)^{n}\left(\delta+\varepsilon_{i}+\gamma_{i}\right)} f_{i j}\left(S_{i}, I_{j}\right)-\omega_{i} I_{i}\right] \\
& \leq \sum_{i=1}^{m}\left[\sum_{j=1}^{m} \frac{\omega_{i} \beta_{i j} c_{i j}\left(S_{i}\right)}{(1+\delta b)^{n}\left(\delta+\varepsilon_{i}+\gamma_{i}\right)} I_{j}-\omega_{i} I_{i}\right] \\
& \leq \sum_{i=1}^{m}\left[\sum_{j=1}^{m} \frac{\omega_{i} \beta_{i j} c_{i j}\left(S_{i}^{0}\right)}{(1+\delta b)^{n}\left(\delta+\varepsilon_{i}+\gamma_{i}\right)} I_{j}-\omega_{i} I_{i}\right] \\
& =\left(\omega_{1}, \ldots, \omega_{m}\right)\left[M^{0} I-I\right] \\
& =\left[\rho\left(M^{0}\right)-1\right]\left(\omega_{1}, \ldots, \omega_{m}\right) I .
\end{aligned}
$$

Thus, under the assumption $R_{0}=\rho\left(M^{0}\right)<1, V_{\mathrm{DFE}}^{\prime} \leq 0$, and $V_{\mathrm{DFE}}^{\prime}=0$ if and only if $I=0$ and $S=S^{0}=\left(S_{1}^{0}, S_{2}^{0}, \ldots, S_{m}^{0}\right)$. Suppose that $\rho\left(M^{0}\right)=1$. Then it follows from the previous that $V_{\mathrm{DFE}}^{\prime}=0$ implies

$$
\left(\omega_{1}, \ldots, \omega_{m}\right) M^{0} I=\left(\omega_{1}, \ldots, \omega_{m}\right) I
$$

Hence, if $S \neq S_{0}$, then $\left(\omega_{1}, \ldots, \omega_{m}\right) M(S)<\left(\omega_{1}, \ldots, \omega_{m}\right) M^{0}=$ $\rho\left(M^{0}\right)\left(\omega_{1}, \ldots, \omega_{m}\right)=\left(\omega_{1}, \ldots, \omega_{m}\right)$ and thus $I=0$ is the only solution of (29). Summarizing the statements, we see that $V_{\mathrm{DFE}}^{\prime}=0$ if and only if $I=0$ or $S=S_{0}$, which implies that the compact invariant subset of the set where $V_{\mathrm{DFE}}^{\prime}=0$ is only the singleton $P_{0}$. Thus, by LaSalle's invariance principle 
[18], it follows that the disease-free equilibrium $E^{0}$ is globally asymptotically stable in $\Gamma_{0}$.

$$
\begin{aligned}
& \text { If } R_{0}=\rho\left(M^{0}\right)>1, \text { then } \\
& \qquad \begin{array}{r}
\left(\omega_{1}, \omega_{2}, \ldots, \omega_{m}\right) M^{0}-\left(\omega_{1}, \omega_{2}, \ldots, \omega_{m}\right) \\
\quad=\left[\rho\left(M^{0}\right)-1\right]\left(\omega_{1}, \omega_{2}, \ldots, \omega_{m}\right)>0,
\end{array}
\end{aligned}
$$

and then, by continuity, we can obtain

$$
V_{\mathrm{DFE}}^{\prime}=\left(\omega_{1}, \ldots, \omega_{m}\right)\left[M^{0} I-I\right]>0,
$$

in a neighborhood of $P_{0}$ in $\Gamma_{0}$; then $P_{0}$ is unstable.

Assume $R_{0}=\rho\left(M^{0}\right)>1$. By the uniform persistence result from [19] and a similar argument as in the proof of [2], the instability of $P_{0}$ implies the uniform persistence of (15). This together with the dissipativity of (15) resulted from the forward invariant and compact property of $\Gamma_{0}$ stated previously, implies which that (15) has an equilibrium in $\Gamma_{0}$, denoted by $\bar{P}^{*}$ (see, e.g., Theorem D.3 in [20]).

In what follows we prove that the endemic equilibrium $\bar{P}^{*}$ of system (15) is globally asymptotically stable when $R_{0}>1$.

Throughout the paper, we denote

$$
H(z)=z-1-\ln z .
$$

Then $H(z) \geq 0$ for $z>0$ and has global minimum at $z=1$.

For convenience of notations, set $\bar{\beta}_{i j}=\beta_{i j} f_{i j}\left(S_{i}^{*}, I_{j}^{*}\right), 1 \leq$ $i, j \leq m$, and

$$
\bar{B}=\left[\begin{array}{cccc}
\sum_{l \neq 1} \bar{\beta}_{1 l} & -\bar{\beta}_{21} & \cdots & -\bar{\beta}_{m l} \\
-\bar{\beta}_{12} & \sum_{l \neq 2} \bar{\beta}_{2 l} & \cdots & -\bar{\beta}_{m 2} \\
\vdots & \vdots & \ddots & \vdots \\
-\bar{\beta}_{1 m} & -\bar{\beta}_{2 m} & \cdots & \sum_{l \neq m} \bar{\beta}_{m l}
\end{array}\right] .
$$

Then, $\bar{B}$ is also irreducible. One knows that the solution space of the linear system

$$
\bar{B} v=0
$$

has dimension 1 and

$$
\left(v_{1}, v_{2}, \ldots v_{m}\right)=\left(C_{11}, \ldots, C_{m m}\right)
$$

gives a base of this space, where $C_{k k}>0, k=1,2, \ldots, m$, is the cofactor of the $k$ th diagonal entry of $\bar{B}$. To get the global stability of $\bar{P}^{*}$, the following assumptions in [15] are proposed:

$$
\begin{aligned}
& \left(A_{4}\right):\left[\varphi_{i}\left(S_{i}\right)-\varphi_{i}\left(S_{i}^{*}\right)\right]\left(S_{i}-S_{i}^{*}\right)<0 \text { for } S_{i} \neq S_{i}^{*}, S_{i} \in\left[0, S_{i}^{0}\right], \\
& \left(A_{5}\right): \text { For } S_{i} \neq S_{i}^{*},\left[\varphi_{i}\left(S_{i}\right)-\varphi_{i}\left(S_{i}^{*}\right)\right] \cdot\left[f_{i i}\left(S_{i}, I_{i}^{*}\right)-f_{i i}\left(S_{i}^{*}, I_{i}^{*}\right)\right]<
\end{aligned}
$$
0 .

$$
\begin{aligned}
& \left(A_{6}\right): \text { For } S_{i}, I_{j}>0, \\
& \left(f_{i i}\left(S_{i}^{*}, I_{i}^{*}\right) f_{i j}\left(S_{i}, I_{j}\right)-f_{i i}\left(S_{i}, I_{i}^{*}\right) f_{i j}\left(S_{i}^{*}, I_{j}^{*}\right)\right) \\
& \quad \cdot\left(\frac{f_{i i}\left(S_{i}^{*}, I_{i}^{*}\right) f_{i j}\left(S_{i}, I_{j}\right)}{I_{j}}-\frac{f_{i i}\left(S_{i}, I_{i}^{*}\right) f_{i j}\left(S_{i}^{*}, I_{j}^{*}\right)}{I_{j}^{*}}\right) \leq 0 .
\end{aligned}
$$

A difficult mathematical question for system (15) is that of whether the endemic equilibrium $\bar{P}^{*}$ is unique when $R_{0}>1$ and whether $\bar{P}^{*}$ is globally asymptotically stable when it is unique. Our main global stability result is given.

Theorem 4. Consider system (15). Assume that $\left(A_{4}\right)-\left(A_{6}\right)$ hold and the matrix $B=\left(\beta_{i j}\right)_{m \times m}$ is irreducible. If $R_{0}>1$, then there is a unique endemic equilibrium $\bar{P}^{*}$ for system (15), and $\bar{P}^{*}$ is globally asymptotically stable in $\Gamma_{0}$.

Proof. We show that $\bar{P}^{*}$ is globally asymptotically stable in $\Gamma_{0}$, which implies that there exists a unique endemic equilibrium.

Consider a Lyapunov function as

$$
\begin{aligned}
V_{\mathrm{EE}}=S_{i}-f_{i i}\left(S_{i}^{*}, I_{i}^{*}\right) \int_{S_{i}^{*}}^{S_{i}} \frac{d \xi}{f_{i i}\left(\xi, I_{i}^{*}\right)} & \\
+(1+\delta b)^{n} & {\left[\sum_{j=1}^{n}\left(y_{i, j}-y_{i, j}^{*}-y_{i, j}^{*} \ln \frac{y_{i, j}}{y_{i, j}^{*}}\right)\right.} \\
& \left.+I_{i}-I_{i}^{*}-I_{i}^{*} \ln \frac{I_{i}}{I_{i}^{*}}\right] .
\end{aligned}
$$

This function has a linear part $V_{\mathrm{EE}}$ expressed by

$$
L_{\mathrm{EE}}=S_{i}+(1+\delta b)^{n}\left[\sum_{j=1}^{n}\left(y_{i, j}-y_{i, j}^{*}\right)+I_{i}-I_{i}^{*}\right] .
$$

First, calculating the derivatives of $L_{\mathrm{EE}}$, we obtain

$$
L_{\mathrm{EE}}^{\prime}=\varphi_{i}\left(S_{i}\right)-(1+\delta b)^{n}\left(\delta+\varepsilon_{i}+\gamma_{i}\right) I_{i} .
$$

Calculating the time derivative of $V_{\mathrm{EE}}$ along the solutions of system (15) and using equilibrium equation (21), we have

$$
\begin{aligned}
V_{\mathrm{EE}}^{\prime}= & L_{\mathrm{EE}}^{\prime}-\frac{f_{i i}\left(S_{i}^{*}, I_{i}^{*}\right)}{f_{i i}\left(S_{i}, I_{i}^{*}\right)} \dot{S}_{i}+(1+\delta b)^{n}\left[\sum_{j=1}^{n} \frac{y_{i, j}^{*}}{y_{i, j}} \dot{y}_{i, j}+\frac{I_{i}^{*}}{I_{i}} \dot{I}_{i}\right] \\
= & \varphi_{i}\left(S_{i}\right)-(1+\delta b)^{n}\left(\delta+\varepsilon_{i}+\gamma_{i}\right) I_{i} \\
& -\left\{\left(\varphi_{i}\left(S_{i}\right)-\sum_{j=1}^{m} \beta_{i j} f_{i j}\left(S_{i}, I_{j}\right)\right) \frac{f_{i i}\left(S_{i}^{*}, I_{i}^{*}\right)}{f_{i i}\left(S_{i}, I_{i}^{*}\right)}\right.
\end{aligned}
$$




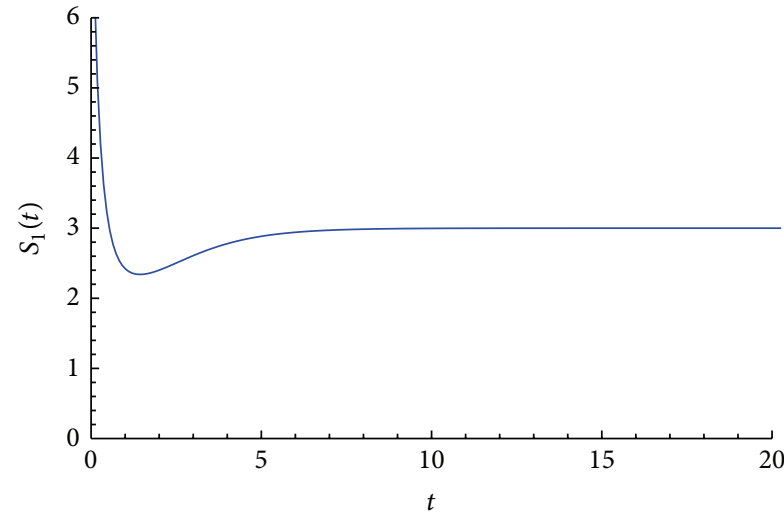

(a)

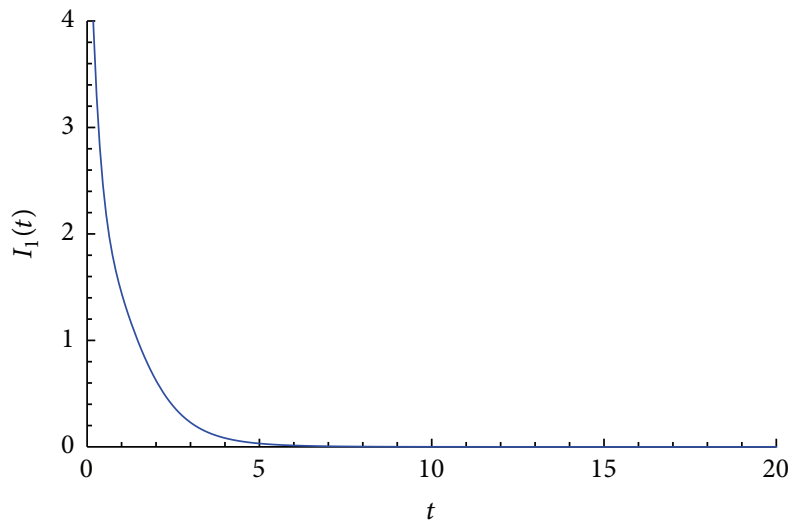

(c)

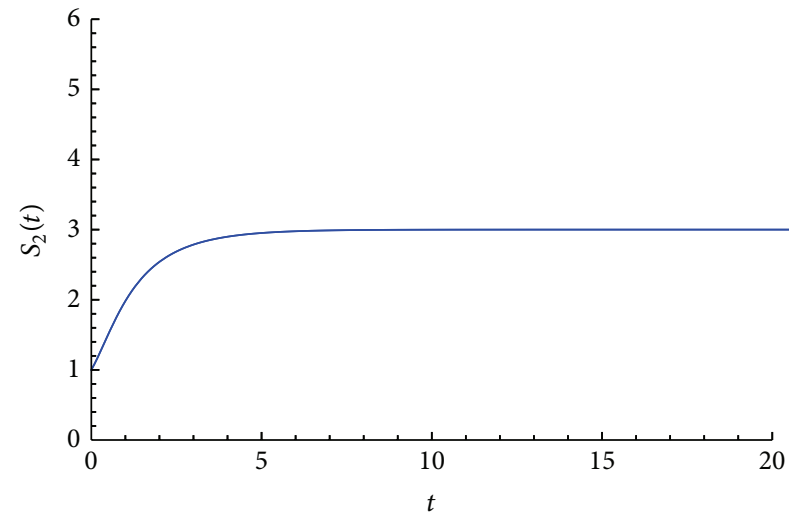

(b)

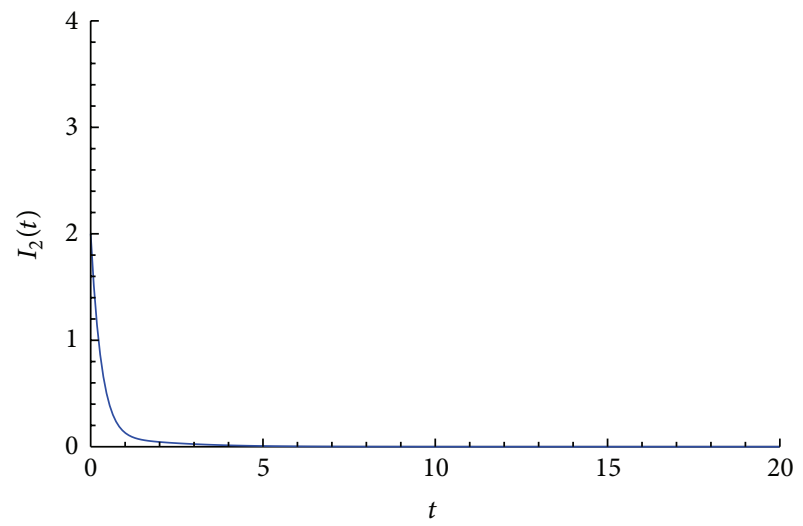

(d)

Figure 1: Trajectories of $S_{1}(t), I_{1}(t), S_{2}(t)$, and $I_{2}(t)$ for $R_{0}=0.051<1$, and $P_{0}=(3,0,0,0,3,0,0,0)$ is globally stable. $S_{1}(t), S_{2}(t), I_{1}(t)$, and $I_{2}(t)$ versus $t$ are illustrated by (a), (b), (c), and (d). Initial values are $S_{1}(0)=9, S_{2}(0)=1, y_{1,1}(0)=2, y_{1,2}(0)=2, y_{2,1}(0)=0, y_{2,2}(0)=$ $0, I_{1}(0)=6$, and $I_{2}(0)=2$.

$$
\begin{gathered}
+(1+\delta b)^{n}\left[\sum_{j=1}^{m} \frac{\beta_{i j} f_{i j}\left(S_{i}, I_{j}\right) y_{i, 1}^{*}}{(1+\delta b)^{n} y_{i, 1}}\right. \\
-\frac{y_{i, 1}^{*}}{\widehat{b}}+\frac{1}{\widehat{b}} \sum_{k=2}^{n} y_{i, k}^{*}\left(\frac{y_{i, k-1}}{y_{i, k}}-1\right) \\
\left.\left.+\frac{y_{i, n} I_{\mathrm{i}}^{*}}{\widehat{b} I_{i}}-\left(\delta+\varepsilon_{i}+\gamma_{i}\right) I_{i}^{*}\right]\right\} \\
\varphi_{i}\left(S_{i}\right)\left(1-\frac{f_{i i}\left(S_{i}^{*}, I_{i}^{*}\right)}{f_{i i}\left(S_{i}, I_{i}^{*}\right)}\right) \\
-\sum_{j=1}^{m} \beta_{i j} f_{i j}\left(S_{i}^{*}, I_{j}^{*}\right) \frac{y_{i, 1}^{*} f_{i j}\left(S_{i}, I_{j}\right)}{y_{i, 1} f_{i j}\left(S_{i}^{*}, I_{j}^{*}\right)} \\
-\frac{(1+\delta b)^{n}}{\widehat{b}} \sum_{k=2}^{n} \frac{y_{i, k}^{*} y_{i, k-1}}{y_{i, k}} \\
+\frac{(1+\delta b)^{n}}{\widehat{b}} n y_{i}^{*}-\frac{(1+\delta b)^{n}}{\widehat{b}} y_{i, n}^{*} \frac{y_{i, n} I_{i}^{*}}{y_{i, n}^{*} I_{i}}
\end{gathered}
$$$$
+(1+\delta b)^{n}\left(\delta+\varepsilon_{i}+\gamma_{i}\right) I_{i}^{*}
$$$$
+\sum_{j=1}^{m} \beta_{i j} f_{i j}\left(S_{i}^{*}, I_{j}^{*}\right) \frac{f_{i j}\left(S_{i}, I_{j}\right) f_{i i}\left(S_{i}^{*}, I_{i}^{*}\right)}{f_{i j}\left(S_{i}^{*}, I_{j}^{*}\right) f_{i i}\left(S_{i}, I_{i}^{*}\right)}
$$$$
-(1+\delta b)^{n}\left(\delta+\varepsilon_{i}+\gamma_{i}\right) I_{i}
$$$$
=\left(\varphi_{i}\left(S_{i}\right)-\varphi_{i}\left(S_{i}^{*}\right)\right)\left(1-\frac{f_{i i}\left(S_{i}^{*}, I_{i}^{*}\right)}{f_{i i}\left(S_{i}, I_{i}^{*}\right)}\right)
$$$$
+\sum_{j=1}^{m} \bar{\beta}_{i j}\left\{n+2-\frac{f_{i i}\left(S_{i}^{*}, I_{i}^{*}\right)}{f_{i i}\left(S_{i}, I_{i}^{*}\right)}-\sum_{k=2}^{n} \frac{y_{i, k}^{*} y_{i, k-1}}{y_{i, k} y_{i, k-1}^{*}}\right.
$$$$
-\frac{y_{i, n} I_{i}^{*}}{y_{i, n}^{*} I_{i}}-\frac{I_{i}}{I_{i}^{*}}-\frac{f_{i j}\left(S_{i}, I_{j}\right) y_{i, 1}^{*}}{f_{i j}\left(S_{i}^{*}, I_{j}^{*}\right) y_{i, 1}}
$$$$
\left.+\frac{f_{i j}\left(S_{i}, I_{j}\right) f_{i i}\left(S_{i}^{*}, I_{i}^{*}\right)}{f_{i j}\left(S_{i}^{*}, I_{j}^{*}\right) f_{i i}\left(S_{i}, I_{i}^{*}\right)}\right\} .
$$ 


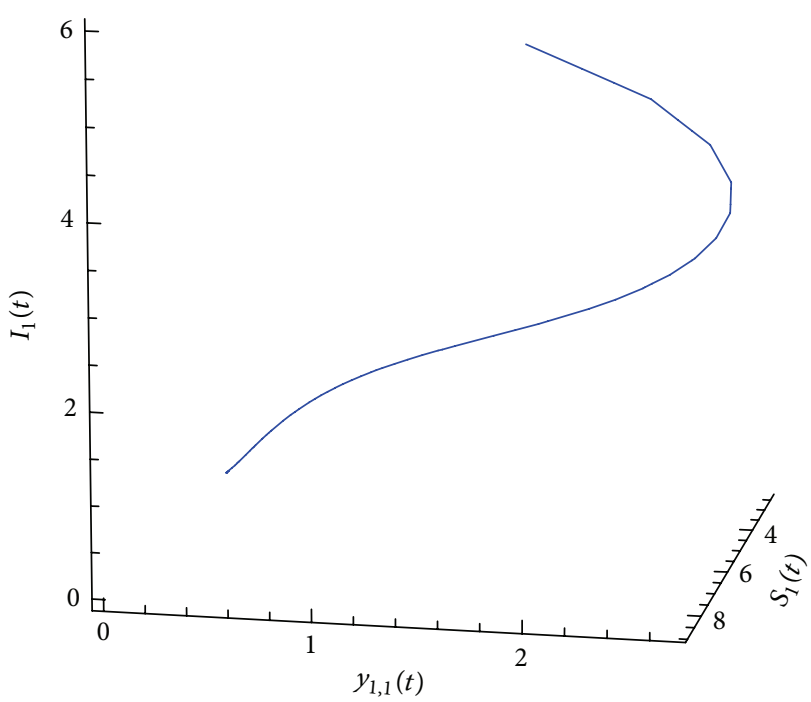

(a)

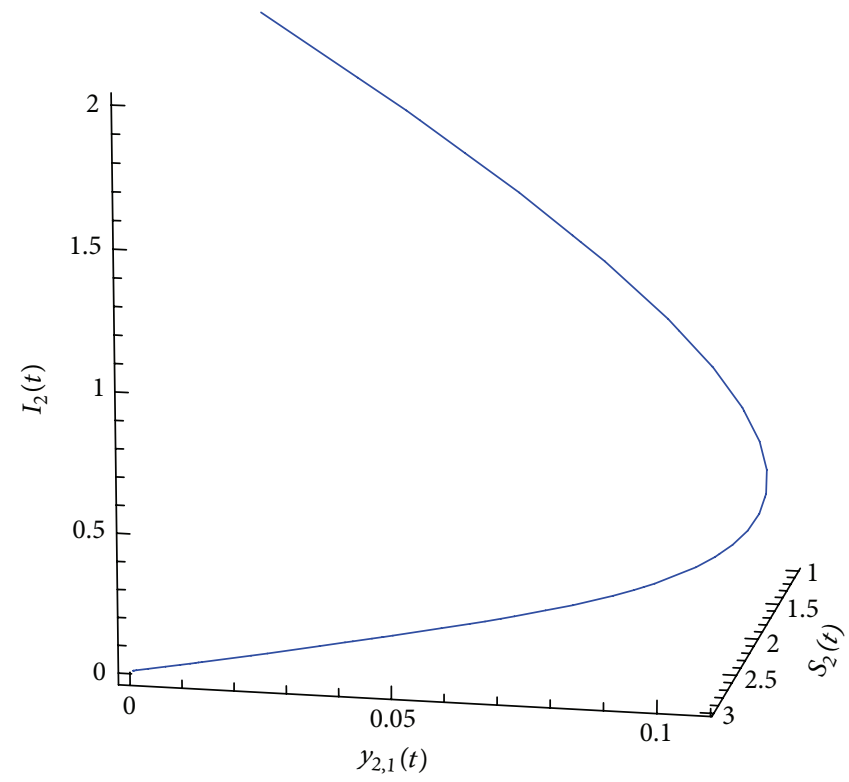

(c)

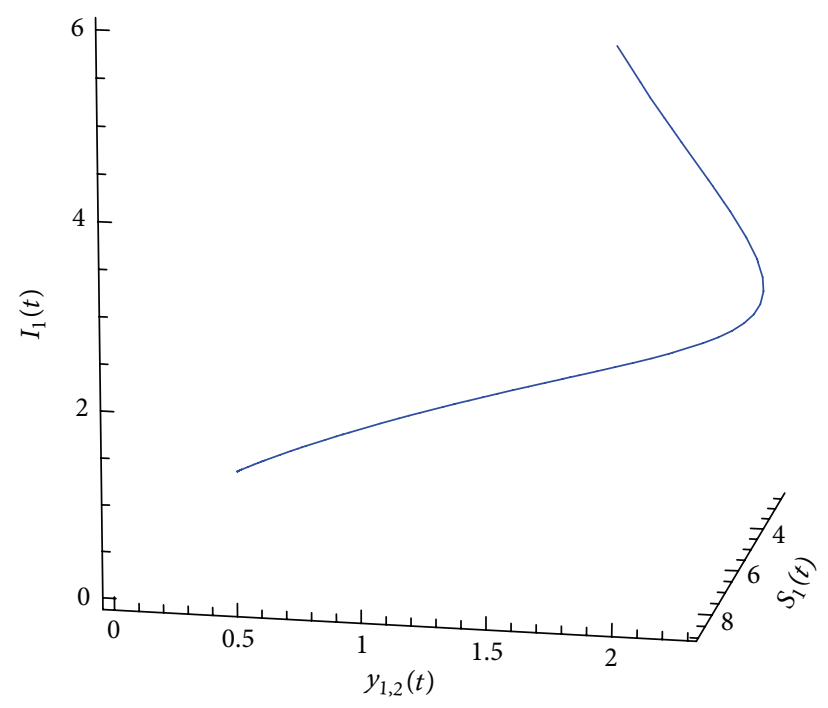

(b)

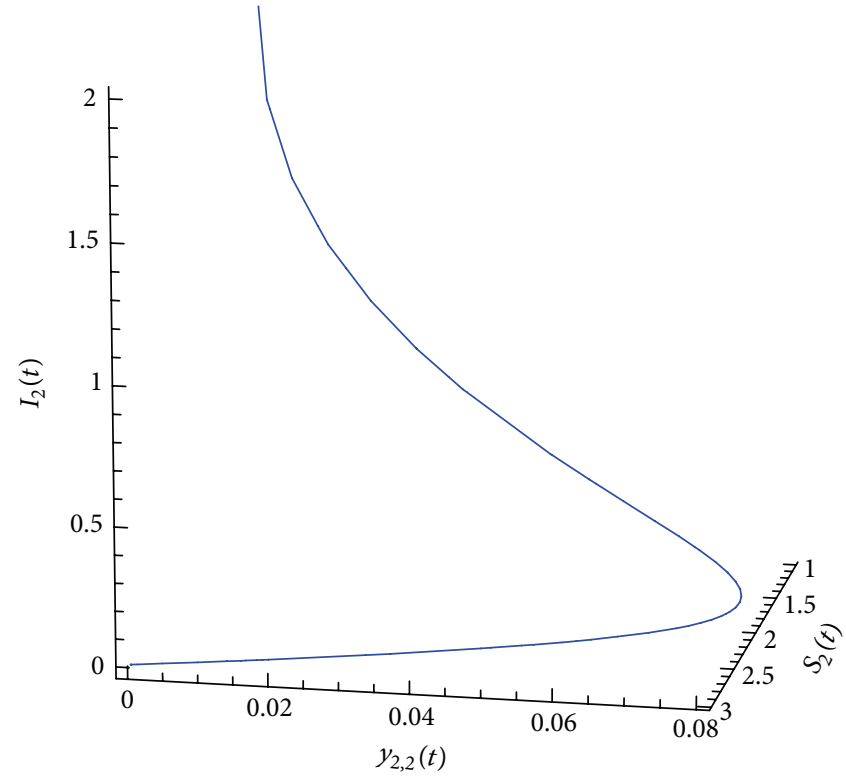

(d)

Figure 2: Numerical simulation of (45) with $R_{0}=0.051<1$; hence $P_{0}=(3,0,0,0,3,0,0,0)$ is globally stable. Graphs (a) and (b) illustrate that $S_{1}(\mathrm{t}), y_{1,1}(t), y_{1,2}(t)$ and $I_{1}(t)$ will eventually towards to steady state. Graphs $(\mathrm{c})$ and $(\mathrm{d})$ illustrate that $S_{2}(t), y_{2,1}(t), y_{2,2}(t)$, and $I_{2}(t)$ will eventually towards to steady state. Initial values are $S_{1}(0)=9, S_{2}(0)=1, y_{1,1}(0)=2, y_{1,2}(0)=2, y_{2,1}(0)=0, y_{2,2}(0)=0, I_{1}(0)=6$, and $I_{2}(0)=2$.

It follows from the assumptions $\left(A_{4}\right)-\left(A_{5}\right)$ that $V_{\mathrm{EE}}^{\prime}$ can be estimated by

$$
\begin{array}{r}
V_{\mathrm{EE}}^{\prime} \leq \sum_{i, j=1}^{m} \bar{\beta}_{i j}\left\{G_{i}\left(I_{i}\right)-G_{j}\left(I_{j}\right)+H\left(\frac{f_{i i}\left(S_{i}^{*}, I_{i}^{*}\right)}{f_{i i}\left(S_{i}, I_{i}^{*}\right)}\right)\right. \\
+H\left(\frac{f_{i j}\left(S_{i}, I_{j}\right) y_{i, 1}^{*}}{f_{i j}\left(S_{i}^{*}, I_{j}^{*}\right) y_{i, 1}}\right)
\end{array}
$$

$$
\begin{aligned}
& +\sum_{k=2}^{n} H\left(\frac{y_{i, k}^{*} y_{i, k-1}}{y_{i, k} y_{i, k-1}^{*}}\right) \\
& +H\left(\frac{y_{i, n} I_{i}^{*}}{y_{i, n}^{*} I_{i}}\right) \\
& +H\left(\frac{I_{j} f_{i i}\left(S_{i}, I_{i}^{*}\right) f_{i j}\left(S_{i}^{*}, I_{j}^{*}\right)}{I_{j}^{*} f_{i i}\left(S_{i}^{*}, I_{i}^{*}\right) f_{i j}\left(S_{i}, I_{j}\right)}\right)
\end{aligned}
$$




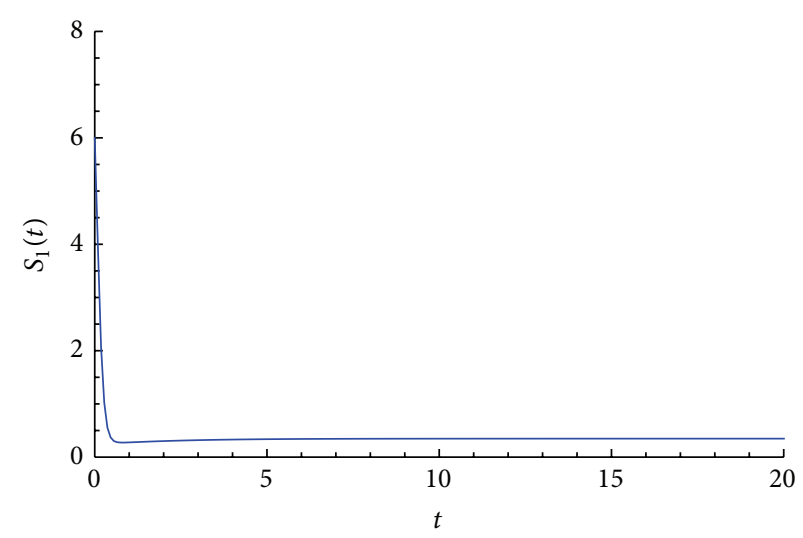

(a)

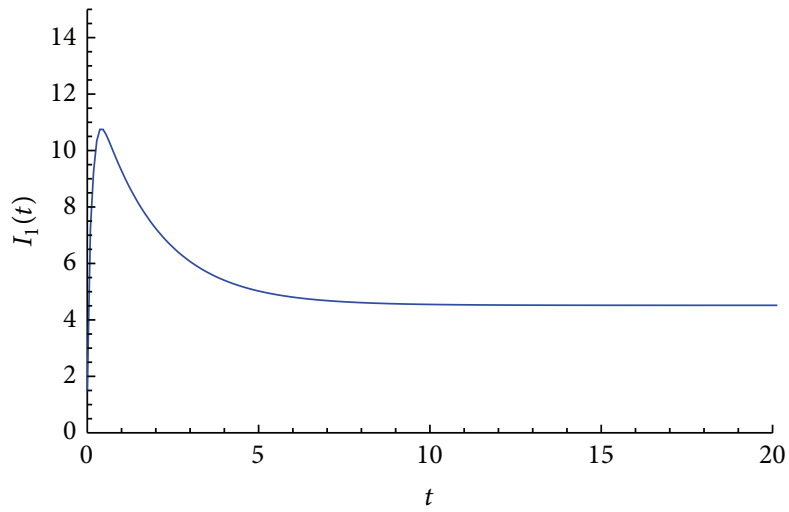

(c)

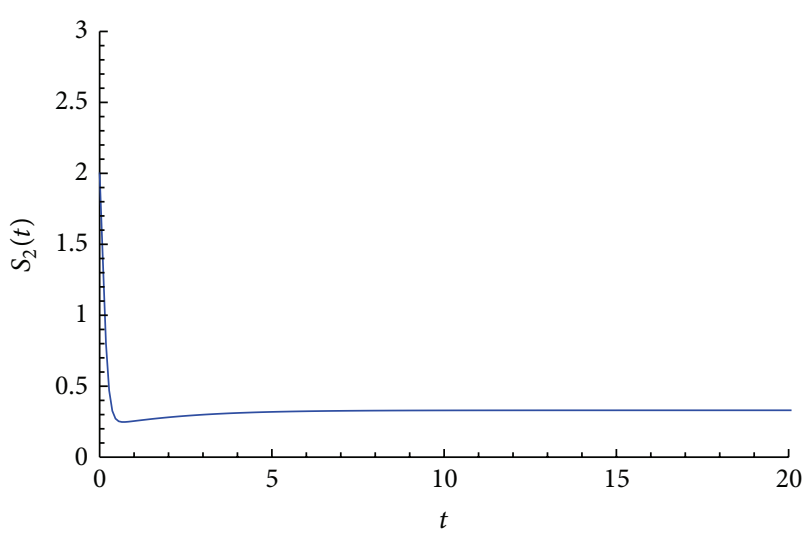

(b)

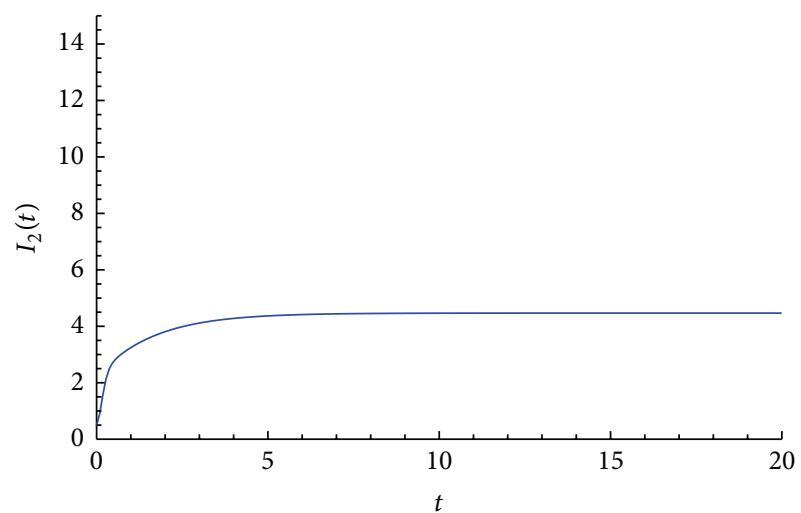

(d)

Figure 3: Trajectories of $S_{1}(t), I_{1}(t), S_{2}(t)$, and $I_{2}(t)$ for $R_{0}=1.67355>1$, and $\bar{P}^{*}=(0.347644,0.0760948,0.0760948,4.51674,0.330353$, $0.0765909,0.0765909,4.4678)$ is globally stable. $S_{1}(t), S_{2}(t), I_{1}(t)$, and $I_{2}(t)$ versus $t$ are illustrated by (a), (b), (c), and (d). Initial values are $S_{1}(0)=6, S_{2}(0)=2, y_{1,1}(0)=3, y_{1,2}(0)=3, y_{2,1}(0)=0.1, y_{2,2}(0)=0.1, I_{1}(0)=1.5$, and $I_{2}(0)=0.5$.

$$
\begin{aligned}
& +\left[\frac{f_{i i}\left(S_{i}^{*}, I_{i}^{*}\right) f_{i j}\left(S_{i}, I_{j}\right)}{f_{i i}\left(S_{i}, I_{i}^{*}\right) f_{i j}\left(S_{i}^{*}, I_{j}^{*}\right)}-1\right] \\
& \left.\cdot\left[1-\frac{I_{j} f_{i i}\left(S_{i}, I_{i}^{*}\right) f_{i j}\left(S_{i}^{*}, I_{j}^{*}\right)}{I_{j}^{*} f_{i i}\left(S_{i}^{*}, I_{i}^{*}\right) f_{i j}\left(S_{i}, I_{j}\right)}\right]\right\} .
\end{aligned}
$$

From the assumption $\left(A_{6}\right)$ and (32), we know that

$$
V_{\mathrm{EE}}^{\prime} \leq \sum_{i, j=1}^{m} \bar{\beta}_{i j}\left\{G_{i}\left(I_{i}\right)-G_{j}\left(I_{j}\right)\right\},
$$

where $G_{i}\left(I_{i}\right)=-I_{i} / I_{i}^{*}+\ln \left(I_{i} / I_{i}^{*}\right)$. if

Obviously, the equalities in (41) and (42) hold if and only

$$
\begin{gathered}
\frac{f_{i i}\left(S_{i}^{*}, I_{i}^{*}\right)}{f_{i i}\left(S_{i}, I_{i}^{*}\right)}=1, \\
\left(1-\frac{f_{i i}\left(S_{i}^{*}, I_{i}^{*}\right)}{f_{i i}\left(S_{i}, I_{i}^{*}\right)}\right)\left[\varphi_{i}\left(S_{i}\right)-\varphi_{i}\left(S_{i}^{*}\right)\right]=0,
\end{gathered}
$$

$$
\begin{aligned}
& {\left[\frac{f_{i j}\left(S_{i}, I_{j}\right) f_{i i}\left(S_{i}^{*}, I_{i}^{*}\right)}{f_{i j}\left(S_{i}^{*}, I_{j}^{*}\right) f_{i i}\left(S_{i}, I_{i}^{*}\right)}-1\right]} \\
& \times\left[1-\frac{f_{i i}\left(S_{i}, I_{i}^{*}\right) f_{i j}\left(S_{i}^{*}, I_{j}^{*}\right) I_{j}}{f_{i i}\left(S_{i}, I_{i}^{*}\right) f_{i j}\left(S_{i}, I_{j}\right) I_{j}^{*}}\right]=0 .
\end{aligned}
$$

That is, $S_{i}=S_{i}^{*}, I_{i}=I_{i}^{*}, i=1,2, \ldots, m$. We can show that $V_{\mathrm{EE}}$ and $\bar{\beta}_{i j}$ satisfy the assumptions of Theorem 3.1 and Corollary 3.3 in [21]. Therefore, the function

$$
L=\sum_{i=1}^{n} v_{i} V_{\mathrm{EE}}
$$

is a Lyapunov function for system (15); namely, $\left.L^{\prime}\right|_{(15)} \leq 0$ for $\bar{P}^{*} \in \Gamma_{0}$. One can only show that the largest invariant subset, where $\left.L^{\prime}\right|_{(15)}=0$, is the singleton $\left\{\bar{P}^{*}\right\}$ by the same 


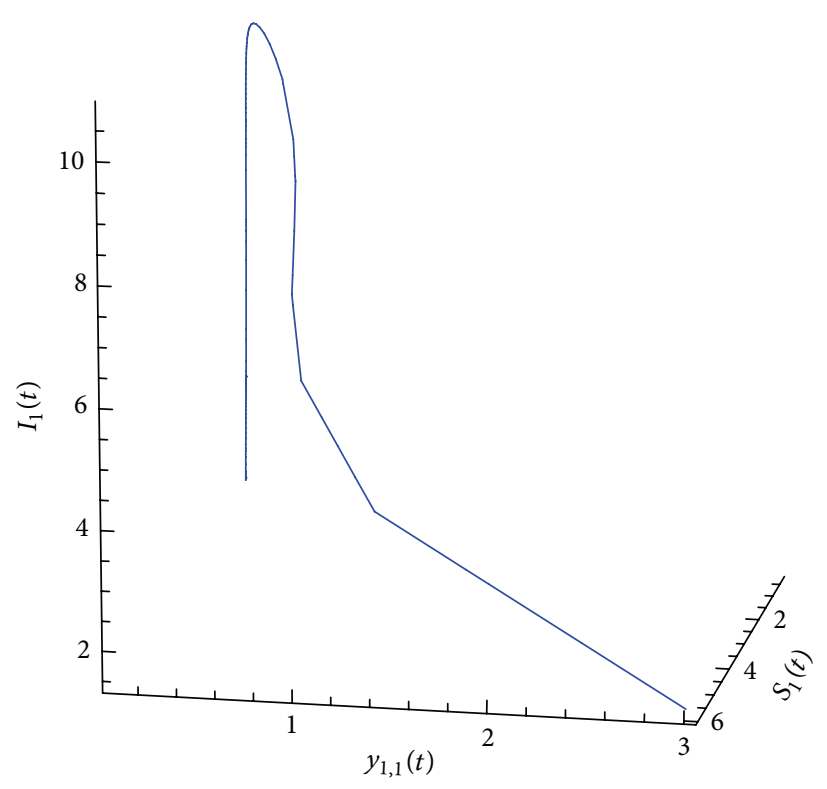

(a)

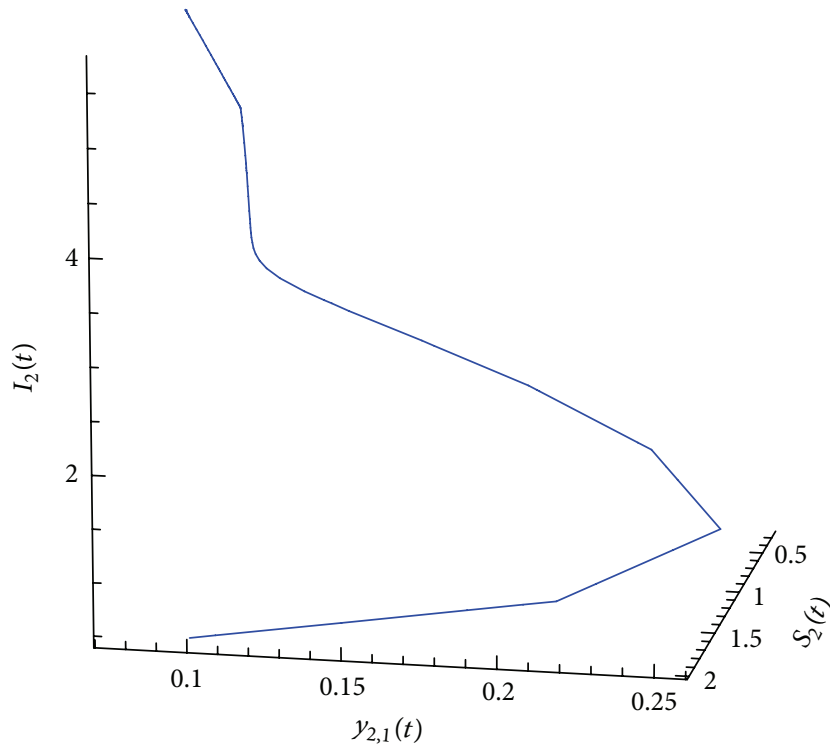

(c)

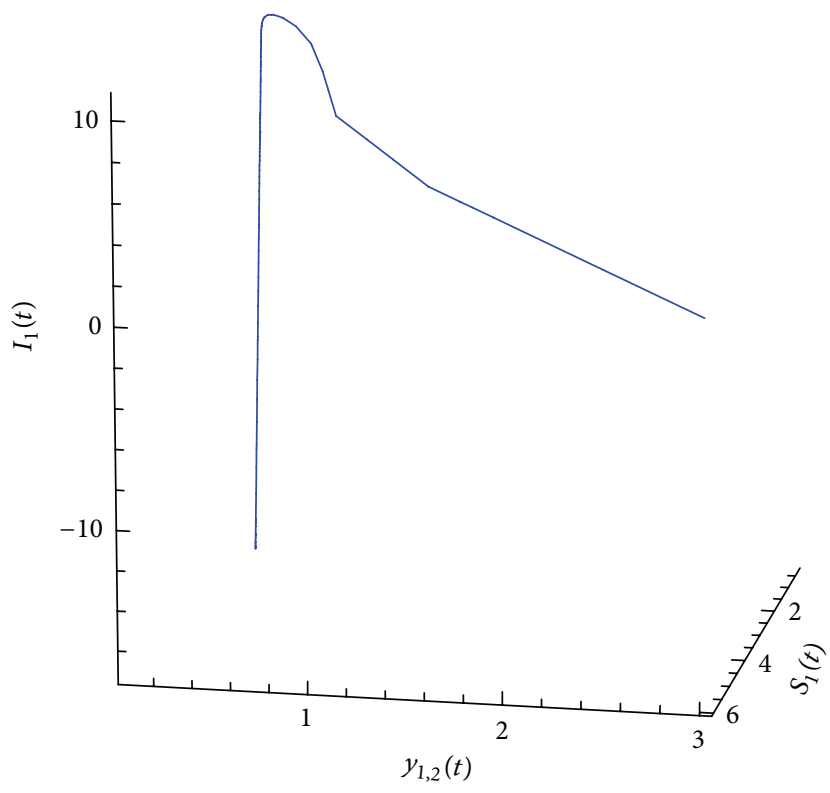

(b)

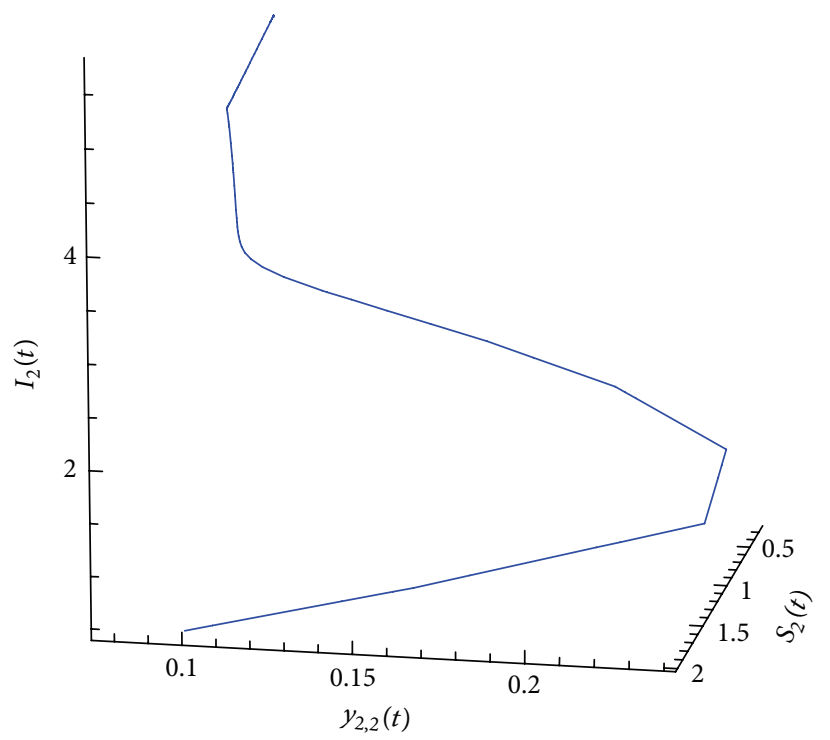

(d)

FIGURE 4: Numerical simulation of $(45)$ with $R_{0}=1.67355>1$; hence $P^{*}=(0.347644,0.0760948,0.0760948,4.51674,0.330353,0.0765909$, $0.0765909,4.4678)$ is globally stable. Graphs (a) and (b) illustrate that $S_{1}(t), y_{1,1}(t), y_{1,2}(t)$, and $I_{1}(t)$ will eventually towards to steady state. Graphs (c) and (d) illustrate that $S_{2}(t), y_{2,1}(t), y_{2,2}(t)$, and $I_{2}(t)$ will eventually towards to steady state. Initial values are $S_{1}(0)=6, S_{2}(0)=$ $2, y_{1,1}(0)=3, y_{1,2}(0)=3, y_{2,1}(0)=0.1, y_{2,2}(0)=0.1, I_{1}(0)=1.5$, and $I_{2}(0)=0.5$.

argument as in $[2-5,13,21]$. By LaSalle's invariance principle, $\bar{P}^{*}$ is globally asymptotically stable in $\Gamma_{0}$. This completes the proof of Theorem 4 .

Remark 5. We show a complete proof for global asymptotic stability of unique endemic equilibrium of system (15). In the case of $f_{i j}\left(S_{i}, I_{j}\right)=S_{i} I_{j}$, system (15) will reduce to the system studied in $[14,22]$. Here Theorem 4 extends related results in $[14,22]$ to a result to a more general case allowing a nonlinear incidence rate. Our result also cover the related results of single group model in [13] for the case of $f(S, I)=f(S) I$.

\section{Numerical Example}

Consider the system (15) when $m=2, n=2, \varphi_{i}\left(S_{i}(t)\right)=$ $3-S_{i}$, and $f_{i j}\left(S_{i}, I_{j}\right)=S_{i} I_{j}, i, j=1,2$. One then has a twogroup model as follows:

$$
\begin{aligned}
S_{1}^{\prime}(t)= & 3-S_{1}-\left[\beta_{11} S_{1}(t) I_{1}(t)+\beta_{12} S_{1}(t) I_{2}(t)\right], \\
y_{1,1}^{\prime}(t)= & \frac{1}{(1+\delta b)^{n}}\left[\beta_{11} S_{1}(t) I_{1}(t)+\beta_{12} S_{1}(t) I_{2}(t)\right] \\
& -\frac{1}{\widehat{b}} y_{1,1}(t),
\end{aligned}
$$




$$
\begin{gathered}
y_{1,2}^{\prime}(t)=\frac{1}{\widehat{b}}\left(y_{1,1}(t)-y_{1,2}(t)\right), \\
I_{1}^{\prime}(t)=\frac{1}{\widehat{b}} y_{1,2}(t)-\left(\delta+\varepsilon_{1}+\gamma_{1}\right) I_{1}(t), \\
S_{2}^{\prime}(t)=3-S_{2}-\left[\beta_{21} S_{2}(t) I_{1}(t)+\beta_{22} S_{2}(t) I_{2}(t)\right], \\
y_{2,1}^{\prime}(t)=\frac{1}{(1+\delta b)^{n}}\left[\beta_{21} S_{2}(t) I_{1}(t)+\beta_{22} S_{2}(t) I_{2}(t)\right] \\
-\frac{1}{\widehat{b}} y_{2,1}(t), \\
y_{2,2}^{\prime}(t)=\frac{1}{\widehat{b}}\left(y_{2,1}(t)-y_{2,2}(t)\right), \\
I_{2}^{\prime}(t)=\frac{1}{\widehat{b}} y_{2,2}(t)-\left(\delta+\varepsilon_{2}+\gamma_{2}\right) I_{2}(t) .
\end{gathered}
$$

If we choose parameters as $\beta_{11}=5 / 24, \beta_{12}=1, \beta_{21}=$ $1 / 36, \beta_{22}=1 / 2, \delta=0.8, \varepsilon_{1}=2, \varepsilon_{2}=2, \gamma_{1}=1 / 4$, and $\gamma_{2}=1 / 4$, we can compute $R_{0}=0.051<1$, and hence $P_{0}=(3,0,0,0,3,0,0,0)$ is the unique equilibrium of system (45) and it is globally stable from Theorem 4 (see Figures 1 and 2).

On the other hand, if $\beta_{i j}$ are chosen as $\beta_{11}=0.7, \beta_{12}=$ $1, \beta_{21}=0.8, \beta_{22}=1, \delta=0.5, \varepsilon_{1}=0.02, \varepsilon_{2}=0.03, \gamma_{1}=0.05$, and $\gamma_{2}=0,05$, we can compute $R_{0}=1.67355>1$, and hence $\bar{P}^{*}=(0.347644,0.0760948,0.0760948,4.51674,0.330353$, $0.0765909,0.0765909,4.4678)$ is the unique equilibrium of system (45) and it is globally stable from Theorem 4 (see Figures 3 and 4).

\section{Acknowledgments}

The authors wish to thank the reviewers for their valuable comments and suggestions that led to truly significant improvement of the paper. J. Wang is supported by the Science and Technology Research Project of the Department of Education of Heilongjiang Province (no. 12531495).

\section{References}

[1] H. R. Thieme, Mathematics in Population Biology, Princeton University Press, Princeton, NJ, USA, 2003.

[2] H. Guo, M. Y. Li, and Z. Shuai, "Global stability of the endemic equilibrium of multigroup SIR epidemic models," Canadian Applied Mathematics Quarterly, vol. 14, no. 3, pp. 259-284, 2006.

[3] H. Guo, M. Y. Li, and Z. Shuai, "A graph-theoretic approach to the method of global Lyapunov functions," Proceedings of the American Mathematical Society, vol. 136, no. 8, pp. 2793-2802, 2008.

[4] H. Shu, D. Fan, and J. Wei, "Global stability of multi-group SEIR epidemic models with distributed delays and nonlinear transmission," Nonlinear Analysis: Real World Applications, vol. 13, no. 4, pp. 1581-1592, 2012.

[5] J. Wang, Y. Takeuchi, and S. Liu, "A multi-group SVEIR epidemic model with distributed delay and vaccination," International Journal of Biomathematics, vol. 5, no. 3, Article ID 1260001, 18 pages, 2012.
[6] P. van den Driessche, L. Wang, and X. Zou, "Modeling diseases with latency and relapse," Mathematical Biosciences and Engineering, vol. 4, no. 2, pp. 205-219, 2007.

[7] A. Berman and R. J. Plemmons, Nonnegative Matrices in the Mathematical Sciences, Academic Press, New York, NY, USA, 1979.

[8] A. Korobeinikov and P. K. Maini, "A Lyapunov function and global properties for SIR and SEIR epidemiological models with nonlinear incidence," Mathematical Biosciences and Engineering, vol. 1, no. 1, pp. 57-60, 2004.

[9] M. Y. Li and J. S. Muldowney, "Global stability for the SEIR model in epidemiology," Mathematical Biosciences, vol. 125, no. 2, pp. 155-164, 1995.

[10] G. Huang and Y. Takeuchi, "Global analysis on delay epidemiological dynamic models with nonlinear incidence," Journal of Mathematical Biology, vol. 63, no. 1, pp. 125-139, 2011.

[11] P. van den Driessche and X. Zou, "Modeling relapse in infectious diseases," Mathematical Biosciences, vol. 207, no. 1, pp. 89103, 2007.

[12] P. van den Driessche, L. Wang, and X. Zou, "Impact of group mixing on disease dynamics," Mathematical Biosciences, vol. 228, no. 1, pp. 71-77, 2010.

[13] S. Liu, S. Wang, and L. Wang, "Global dynamics of delay epidemic models with nonlinear incidence rate and relapse," Nonlinear Analysis: Real World Applications, vol. 12, no. 1, pp. 119-127, 2011.

[14] Z. Yuan and X. Zou, "Global threshold property in an epidemic model for disease with latency spreading in a heterogeneous host population," Nonlinear Analysis: Real World Applications, vol. 11, no. 5, pp. 3479-3490, 2010.

[15] R. Sun and J. Shi, "Global stability of multigroup epidemic model with group mixing and nonlinear incidence rates," Applied Mathematics and Computation, vol. 218, no. 2, pp. 280286, 2011.

[16] R. K. Miller, Nonlinear Volterra Integral Equations, W. A. Benjamin, New York, NY, USA, 1971.

[17] P. van den Driessche and J. Watmough, "Reproduction numbers and sub-threshold endemic equilibria for compartmental models of disease transmission," Mathematical Biosciences, vol. 180, no. 1-2, pp. 29-48, 2002.

[18] J. P. Lasalle, The Stability of Dynamical Systems, Regional Conference Series in Applied Mathematics, SIAM, Philadelphia, Pa, USA, 1976

[19] H. I. Freedman, M. X. Tang, and S. G. Ruan, "Uniform persistence and flows near a closed positively invariant set," Journal of Dynamics and Differential Equations, vol. 6, no. 4, pp. 583-600, 1994.

[20] H. L. Smith and P. Waltman, The Theory of the Chemostat: Dynamics of Microbial Competition, Cambridge University Press, Cambridge, UK, 1995.

[21] M. Y. Li and Z. S. Shuai, "Global-stability problem for coupled systems of differential equations on networks," Journal of Differential Equations, vol. 248, no. 1, pp. 1-20, 2010.

[22] Z. Shuai and P. van den Driessche, "Impact of heterogeneity on the dynamics of an SEIR epidemic model," Mathematical Biosciences and Engineering, vol. 9, no. 2, pp. 393-411, 2012. 


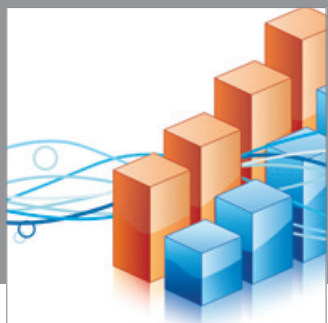

Advances in

Operations Research

mansans

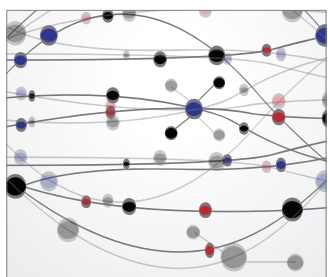

The Scientific World Journal
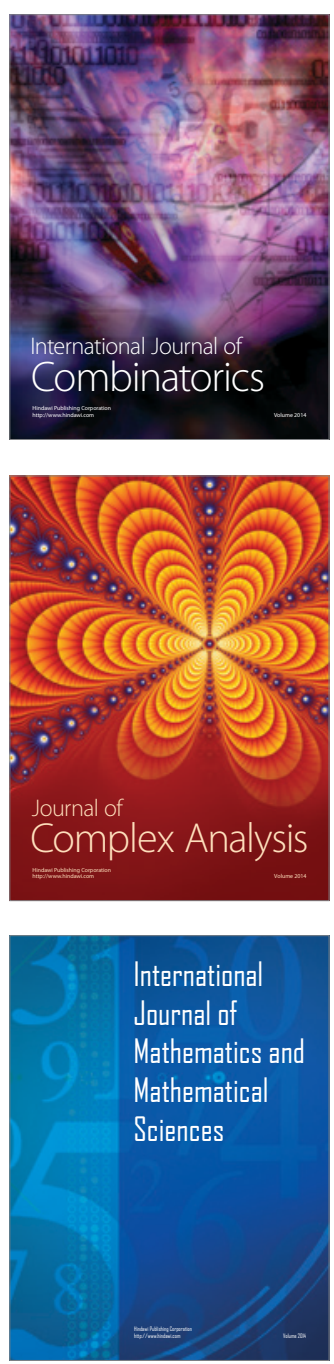
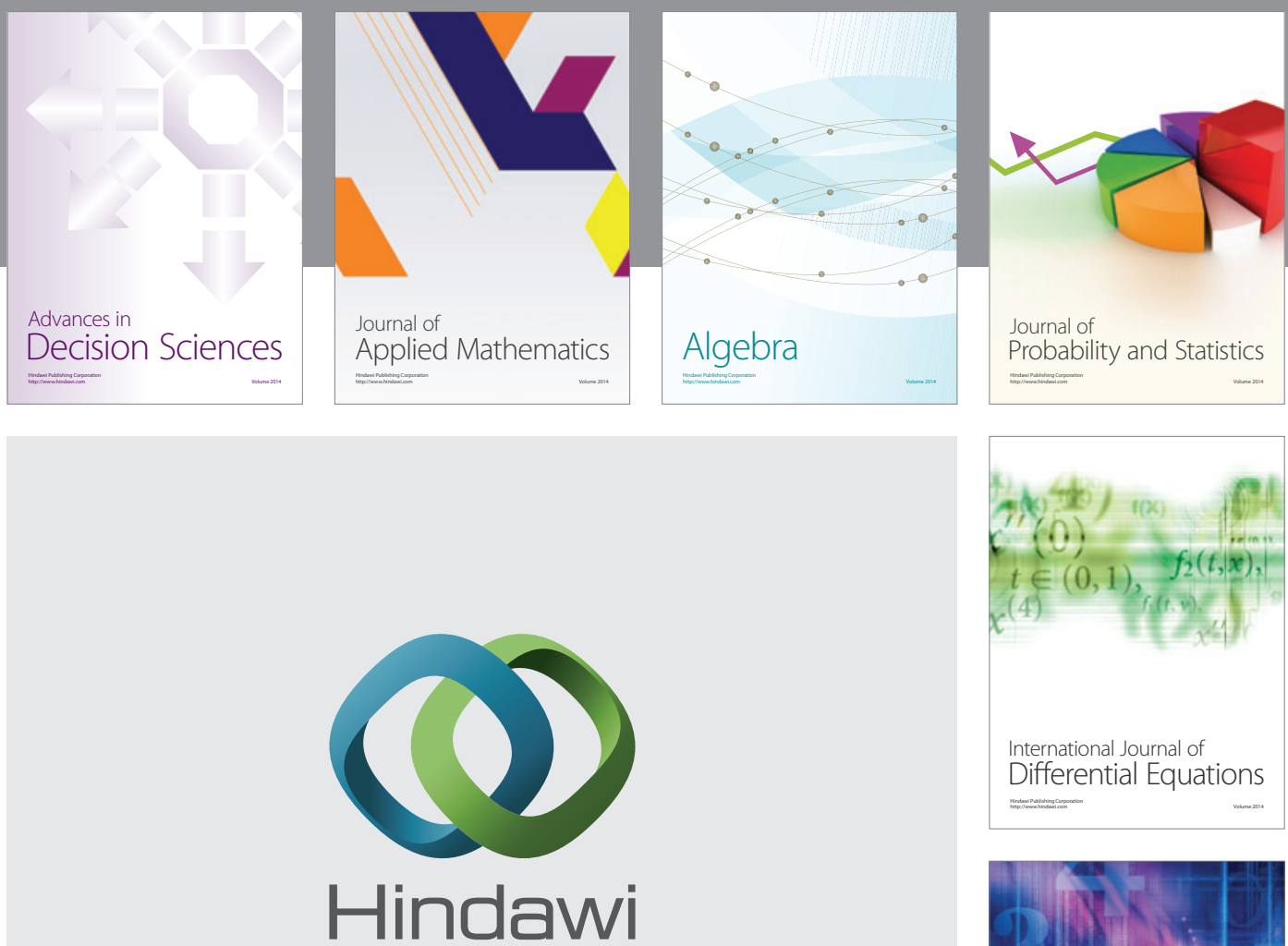

Submit your manuscripts at http://www.hindawi.com
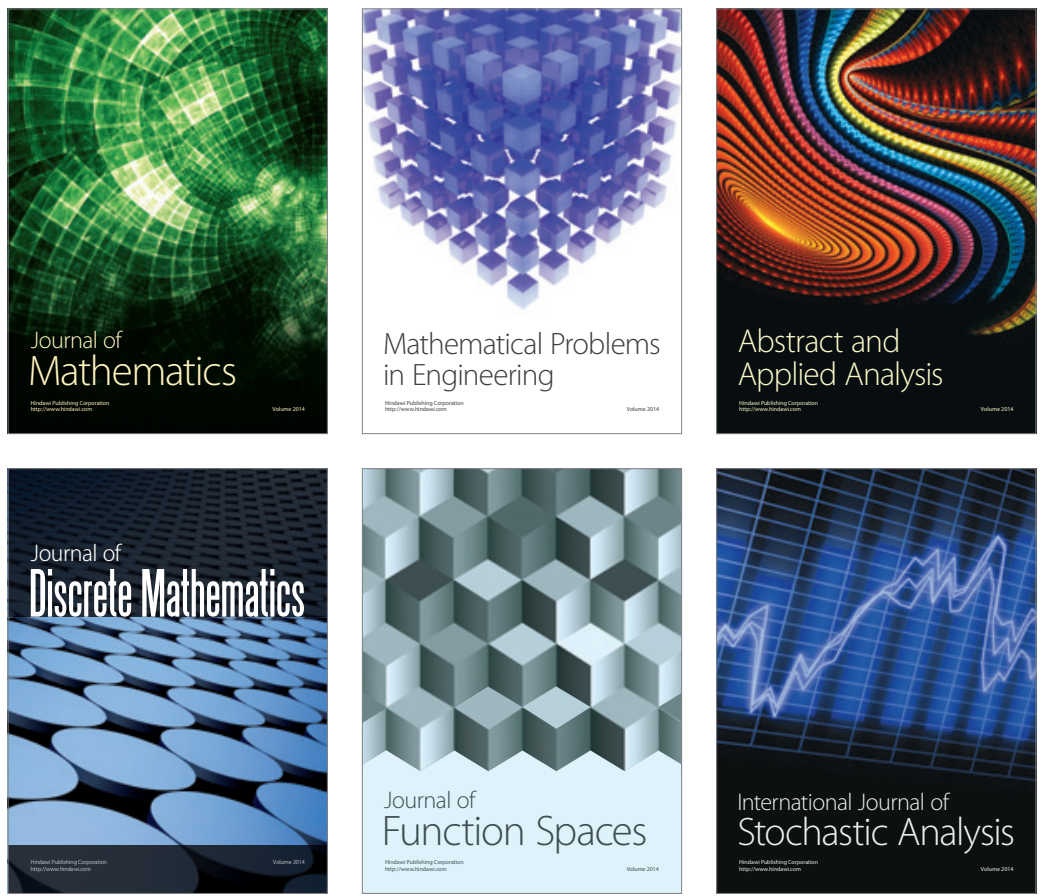

Journal of

Function Spaces

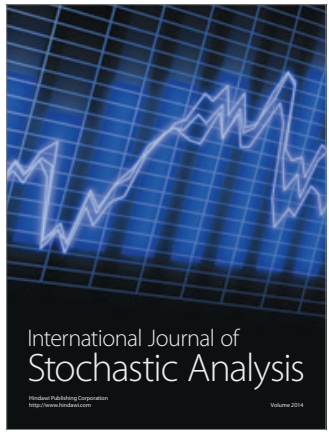

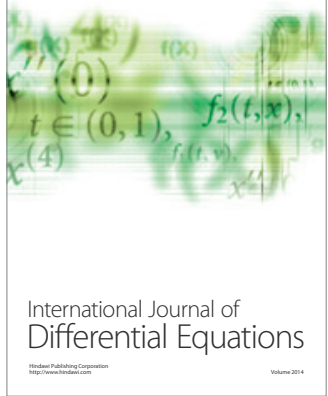
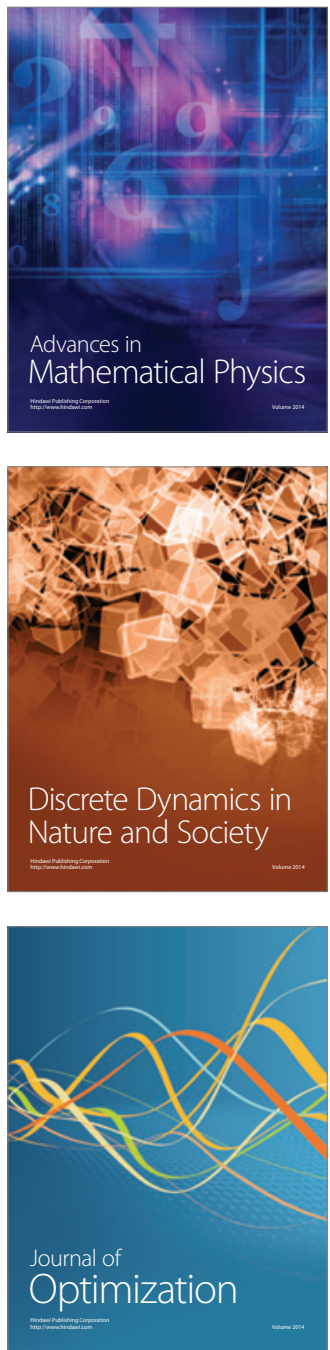